\title{
Spirulina, exercício e controle da glicemia em ratos diabéticos
}

\author{
Spirulina, exercise and serum glucose control in diabetic rats
}

Leandro Pereira de Moura', André Luiz Demantova Gurjão', José Claudio

Jambassi Filho', Julio Mizuno', Clara Suemi', Maria Alice Rostom de Mello'

\section{RESUMO}

Objetivo: O objetivo do presente estudo foi analisar o efeito da Spirulina e/ou do treinamento físico na homeostase glicêmica de ratos diabéticos. Materiais e métodos: Ratos Wistar diabéticos aloxânicos foram separados em quatro grupos: diabético controle (DC); diabético Spirulina (DS); diabético exercício (DE); diabético Spirulina exercício (DSE). Resultados: Não foram observadas diferenças significativas entre os grupos para: peso corporal, ingestão alimentar, tolerância à glicose, tolerância à insulina, concentrações de lactato sanguíneo durante teste de esforço. Para as concentrações de insulina, o grupo DS apresentou valor significativamente menor quando comparado ao grupo DC (pâncreas) e DE e DES (soro). Conclusão: Os protocolos de exercício e de suplementação com Spirulina utilizados no presente estudo não foram suficientes para promover melhora na homeostase glicêmica de ratos diabéticos. Arq Bras Endocrinol Metab. 2012;56(1):25-32

\section{Descritores}

Exercício; diabetes; Spirulina; ratos

\section{ABSTRACT}

Objective: The objective of this study was to analyze the effect of Spirulina and/or exercise training in the control of serum glucose homeostasis in diabetic rats. Materials and methods: Young Wistar rats were induced to diabetes by intravenous alloxan administration and separated into four groups: diabetic control (DC), diabetic Spirulina (DS), diabetic exercise (DE) and diabetic exercise Spirulina (DES). Results: There were no differences between groups with respect to: body weight, food intake, glucose tolerance, insulin tolerance and blood lactate concentrations during a swimming effort test. DS group showed lower insulin concentrations when compared with DC (pancreas) and DE and DES (serum). Conclusion: The protocols of exercise and supplementation with Spirulina used in the present study were not able to improve serum glucose homeostasis in diabetic rats. Arq Bras Endocrinol Metab. 2012;56(1):25-32

Departamento de Educação Física, Instituto de Biociências, Universidade Estadual Paulista (Unesp), Rio Claro, SP, Brasil
Correspondência para: Leandro Pereira de Moura Departamento de Educação Física, Instituto de Biociências,

Universidade Estadual Paulista Av. 24 A, 1515

13506-900 - Rio Claro, SP, Brasil leandropereiram@hotmail.com

Recebido em 25/Abr/2011 Aceito em 29/Jan/2012

\section{INTRODUÇÃO}

$\mathrm{O}$ diabetes melito (DM) é um problema de saúde pública mundial. Estima-se que existam mais de $150 \mathrm{mi}^{-}$ lhões de pessoas com essa doença no mundo, e projeções da Organização Mundial da Saúde (OMS) para 2025 sugerem que esse número possa chegar a 300 milhões (1).

O DM é caracterizado pela redução da secreção (diabetes melito tipo l (DMl) - insulino-dependente) ou da ação da insulina (diabetes melito tipo 2 (DM2) - não insulino-dependente), acarretando hiperglicemia, além de alterações no metabolismo das proteínas e dos lipídeos (2). As consequências para o organismo são variadas, incluindo hipertensão, nefropatias, retinopatias e cardiopatias (3). Em consequência dos efeitos colaterais causados por agentes terapêuticos usados para o tratamento dessa patologia, como os hipoglicemiantes orais e a insulina (4), atualmente existe grande interesse nas ervas medicinais (5), como hipoglicemiantes naturais (6), entre elas a Spirulina (7).

A Spirulina é uma alga verde-azulada de forma helicoidal, com comprimento de 0,2-0,5 mm (8), 
que contém $65 \%$ a $70 \%$ de proteína, com base no peso seco, sendo superior a qualquer outra fonte natural de alimento, além de conter os oito aminoácidos essenciais (9). Outra vantagem dessa alga é sua reprodução rápida, dividindo-se três vezes ao dia. Por exemplo, em uma mesma área, ela pode produzir 125 vezes mais proteína do que o cultivo de milho, 70 vezes mais a criação de peixes ou 600 vezes mais a criação de gado (10).

De acordo com avaliações prévias realizadas com seres humanos, apesar de a Spirulina ter digestibilidade um pouco reduzida, ela apresenta boa aceitação e não contém efeitos tóxicos aparentes (11). Em estudos com animais de laboratório, especialmente ratos, constataram-se boa aceitação, uma adequada digestibilidade, um bom suplemento durante a gestação (12), bem como a ausência de qualquer efeito deletério sobre marcadores de crescimento das crias e tóxicos aparentes (13). Adicionalmente, outros estudos envolvendo animais e humanos verificaram que sua administração pode exercer efeitos benéficos no tratamento de algumas doenças, como câncer $(7,14)$, desnutrição $(7,15)$, obesidade $(7,16)$, hipercolesterolemia $(7,9)$, hipertensão arterial $(7,17)$, DM (7), entre outras.

As frações hidro e lipossolúveis da Spirulina são efetivas em diminuir os níveis de glicose sérica de ratos em jejum $(18,19)$. Segundo estudo realizado por Layam e Reddy (20), essa alga, administrada na concentração de $15 \mathrm{mg} / \mathrm{kg}$ corporal, pode aumentar os níveis séricos de insulina em ratos insulino-dependentes.

Outro fator bastante recomendado para o controle do quadro glicêmico de indivíduos diabéticos, melhorando a tolerância à glicose e reduzindo a resistência à insulina, é a prática de exercícios físicos (21). Dessa forma, a associação da suplementação da Spirulina com a prática de exercícios físicos pode ser uma estratégia para o controle da DM ainda não estudada na literatura. Assim, o objetivo do presente estudo foi analisar o efeito da Spirulina e/ou do treinamento no controle da homeostase glicêmica de ratos com diabetes insulino-dependente.

\section{MATERIAIS E MÉTODOS}

\section{Animais}

Para o desenvolvimento do presente trabalho, foram utilizados ratos, jovens (60 dias no início do experimento), da linhagem Wistar. Os animais, provenientes do Biotério Central da Universidade Estadual Paulista (Unesp), Botucatu, SP, Brasil, foram mantidos no Biotério do Departamento de Educação Física da Unesp, Rio Claro, SP, Brasil. Os ratos foram alimentados com dietas semipurificadas, preparadas pelos pesquisadores, e água "ad libitum", e mantidos em gaiolas plásticas coletivas (cinco por gaiola) à temperatura ambiente de $22 \pm 1^{\circ} \mathrm{C}$ e foto período de 12 horas de claro e 12 de escuro.

\section{Aspectos éticos}

O experimento foi realizado de acordo com a legislação brasileira sobre o uso científico de animais (lei $\mathrm{n}^{\circ}$ 11.794, de 8 de outubro de 2008). O protocolo foi aprovado pela Comissão de Ética no Uso de Animal (CEUA), do Instituto de Biociências, da Universidade Estadual Paulista (Unesp), Rio Claro, SP, Brasil, protocolo n. 5111 de 20 de agosto de 2009.

\section{Grupos experimentais}

Os animais, depois de confirmada a instalação do diabetes, foram distribuídos em quatro grupos experimentais e tratados dos 70 aos 112 dias de idade. Grupos: diabético controle (DC): ratos diabéticos sedentários que não receberam tratamento; diabético Spirulina (DS): ratos diabéticos sedentários tratados com Spirulina; diabético exercício (DE): ratos diabéticos que foram submetidos ao protocolo de treinamento físico sem serem tratados com Spirulina; diabético Spirulina exercício (DSE): ratos diabéticos que foram submetidos ao protocolo de treinamento físico e tratados com Spirulina.

\section{Indução do diabetes}

Para a obtenção do diabetes experimental, os ratos, depois de permanecerem 12 horas em jejum, foram anestesiados com $\mathrm{CO}_{2}$ e receberam aloxana $(32 \mathrm{mg} / \mathrm{kg}$ de peso corporal), dissolvida em tampão citrato $0,01 \mathrm{M}$, pH 4,5 e injetada via veia peniana dorsal (22). Logo após a administração da droga, os animais receberam solução de água com $15 \%$ de glicose devido ao efeito hipoglicêmico decorrente da droga (23). Foram considerados diabéticos os animais que apresentaram glicemia de jejum igual ou superior a $126 \mathrm{mg} / \mathrm{dL}$, de acordo com a Associação Americana de Diabetes (24), duas semanas após a administração de aloxana, por meio de determinação dos níveis glicêmicos pelo método da glicose-oxidase (Laborlab ${ }^{\circledast}$, Guarulhos, SP, Brasil). 


\section{Dietas}

Foram utilizadas dietas isocalóricas $(3.028 \mathrm{kcal} / \mathrm{kg})$ e normoproteicas semipurificadas: dieta controle (contendo $17 \%$ de proteína na forma de caseína, de acordo com as recomendações do American Institute of Nutrition, AIN-G93 (25), para manter os animais dos grupos controle (DC) e exercício (DE) e dieta Spirulina (contendo 17\% de proteína na forma de Spirulina), para manter os ratos dos grupos Spirulina (DS e DSE). As dietas controle e Spirulina tiveram a seguinte composição (\%): amido de milho $=39,7$; dextrina $=$ 13,$2 ;$ sacarose $=10$; óleo de soja $=7$; microcelulose $=5$; mistura de sais minerais $=3,5$; mistura de vitaminas 1,0 . A composição detalhada das misturas de sais minerais e de vitaminas foi descrita de acordo com o American Institute of Nutrition, AIN-G93 (25).

\section{Adaptação ao meio líquido}

A adaptação consistiu em manter o animal em contato com água rasa durante uma semana. Isso visou reduzir o estresse dos animais e adaptá-los tanto ao protocolo de treinamento físico quanto ao teste de esforço, ambos realizados na água à temperatura de $31 \pm 1^{\circ} \mathrm{C}(26)$.

\section{Treinamento físico}

$\mathrm{O}$ treinamento de natação consistiu de seis semanas em tanques coletivos $(100 \mathrm{~cm} \mathrm{x} 80 \mathrm{~cm} \mathrm{x} 80 \mathrm{~cm})$, contendo água a $31 \pm 1^{\circ} \mathrm{C}, 5$ dias por semana, suportando sobrecarga, atada ao tórax do animal, equivalente a 3,5\% do peso corporal. Tal protocolo foi selecionado por representar exercício aeróbico de intensidade leve/moderada para ratos diabéticos (27).

\section{Avaliações gerais}

Todos os animais tiveram peso corporal e ingestão alimentar registrados uma vez por semana.

\section{Teste de tolerância à glicose}

O teste de tolerância à glicose (TTGo) foi realizado com os animais na última semana do experimento, após 12 horas de jejum. Uma primeira coleta de sangue foi feita por meio de corte na extremidade da cauda do animal (tempo 0). Em seguida, uma solução de glicose a $80 \%$, na dose de $2 \mathrm{~g} / \mathrm{kg}$ de peso corporal, foi administrada aos ratos por sonda gástrica de polietileno. Para determinar as concentrações de glicose, amostras de sangue foram coletadas após 30, 60 e 120 minutos com capilares heparinizados e calibrados para $25 \mu \mathrm{L}$. Um único corte na extremidade da cauda do animal foi suficiente para a coleta de todas as amostras sanguíneas. As concentrações de glicose sanguínea foram determinadas pelo método glicose-oxidase $\left(\right.$ Laborlab $^{\circledR}$, Guarulhos - SP/Brasil). Os resultados foram analisados por meio da determinação das áreas sob as curvas de glicose sérica durante o teste pelo método trapezoidal (28), utilizando-se o software ORIGIN.

\section{Teste de tolerância à insulina}

A sensibilidade à insulina foi avaliada pelo teste de tolerância à insulina (TTI) 48 horas após o TTGo. A primeira coleta de sangue foi feita através de corte na extremidade da cauda do animal (tempo 0). Em seguida, uma solução de insulina cristalina "LILLY U 40", na dose de $30 \mathrm{mU} / 100 \mathrm{~g}$ de peso corporal, foi administrada via subcutânea. Amostras de sangue foram coletadas após 30, 60 e 120 minutos com capilares heparinizados e calibrados para $25 \mu \mathrm{L}$, visando à determinação das concentrações de glicose, utilizando kits comerciais (Laborlab $^{\circledR}$, Guarulhos - SP, Brasil). Um único corte na extremidade da cauda do animal foi suficiente para a coleta de todas as amostras sanguíneas. A taxa de remoção de glicose (Kitt) expressa em \%/minuto foi calculada pela fórmula $(0,0693 / \mathrm{t} / 2)$ X100. A glicose sanguínea $(t / 2)$ foi calculada pela curva de análise dos mínimos quadrados dos teores de glicose no sangue nos momentos após a administração de insulina em que a glicose sérica decaiu (29).

\section{Teste de esforço}

Quarenta e oito horas após o TTI todos os animais foram submetidos a uma sessão de 20 minutos de natação, suportando sobrecarga de chumbo atada ao tórax equivalente a $3,5 \%$ do peso corporal, com coleta de sangue $(25 \mu \mathrm{L})$ a cada cinco minutos, a partir de um pequeno corte na extremidade distal da cauda do animal, para a dosagem de lactato. As concentrações de lactato sanguíneo foram determinadas pelo método enzimático (30). Os animais sedentários foram adaptados durante uma semana para redução do estresse.

\section{Obtenção de material biológico}

Ao final do experimento, 48 horas depois da última avaliação in vivo e em repouso, os animais foram exsanguinados depois de serem anestesiados com $\mathrm{CO}_{2}$, sendo o sangue coletado para a separação do soro, vi- 
sando à dosagem de glicose, pelo método da glicose oxidase, utilizando kits comerciais (Laborlab ${ }^{\circledast}$, Guarulhos - SP, Brasil) e insulina por método de radioimunoensaio (31). O pâncreas foi removido e alíquotas de $0,2 \mathrm{~g}$ foram colocadas em frascos contendo $2 \mathrm{~mL}$ de $\mathrm{HCl} \mathrm{IN}$. Essas alíquotas foram incubadas em estufa a $40^{\circ} \mathrm{C}$ durante 24 horas. O material assim extraído do pâncreas foi diluído 1:5000 em soro-albumina bovina a $0,25 \%$ em tampão borato $\mathrm{pH} 5,5$, conforme Carpinelli (32). O teor de insulina das amostras foi dosado pelo método do radioimunoensaio (31).

\section{Análise estatística}

Os resultados foram expressos como média \pm desvio-padrão. O teste de Shapiro Wilk foi utilizado para análise da distribuição dos dados. ANOVA one-way foi empregada para as comparações entre grupos referentes à massa corporal no início e no final do estudo, à ingestão alimentar ao final do estudo, às áreas sob as curvas da glicose durante o teste de tolerância à glicose, à taxa de remoção de glicose sérica durante o teste de tolerância à insulina e às concentrações de insulina pancreática e sérica ao final do experimento. ANOVA 4 X 5 para medidas repetidas foi empregada para as comparações das concentrações de lactato sanguíneo no teste de esforço entre os grupos (DC; DS; DE e; DSE) e os momentos $(0 ; 5 ; 10 ; 15$ e; 20 minutos). Em ambas as análises, o teste post hoc de Scheffé foi utilizado para comparações múltiplas quando necessário. O nível de significância adotado para todas as análises foi de $\mathrm{p}<0,05$. Os procedimentos estatísticos foram realizados no programa SPSS, versão 10.0.

\section{RESULTADOS}

Os resultados da massa corporal média de cada grupo estão demonstrados na figura 1. Todos os grupos reduziram significativamente a massa corporal no final dos 44 dias de treinamento em relação aos valores pré-intervenção. A análise estatística não demonstrou diferença significante quando comparados todos os grupos quanto à ingestão alimentar ao final do experimento (Figura 2).

Não foram constatadas diferenças significativas para as concentrações de lactato sanguíneo entre os grupos durante teste de esforço (Figura 3). As áreas sob as curvas de glicose sérica para os diferentes grupos, obtidas durante o TTGo, são apresentadas na figura 4. Não foram observadas diferenças significativas entre os grupos.

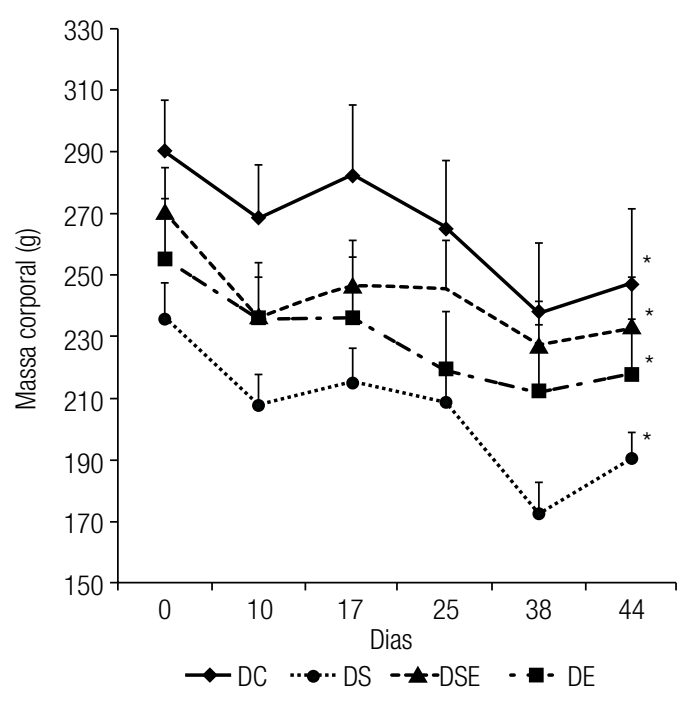

Figura 1. Peso corporal dos animais ao longo de todo o experimento para os grupos diabético controle (DC; $n=7)$; diabético Spirulina(DS; $n=10)$; diabético exercício (DE; $n=8)$; diabético Spirulina exercício (DSE; $n=11)$. Os dados são expressos em média \pm desvio-padrão. *: diferente da situação inicial.

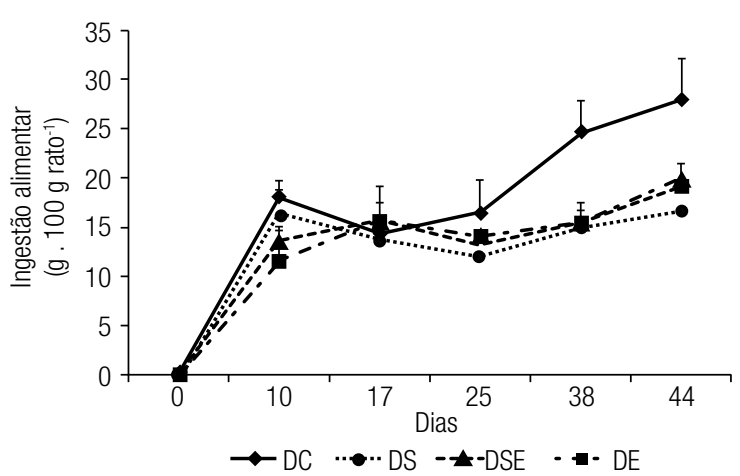

Figura 2. Ingestão alimentar ao longo de todo o experimento para os grupos diabético controle (DC; $n=7$ ); diabético Spirulina (DS; $n=10)$; diabético exercício (DE; $n=8)$; diabético Spirulina exercício (DSE; $n=11$ ). Os dados são expressos em média \pm desvio-padrão.

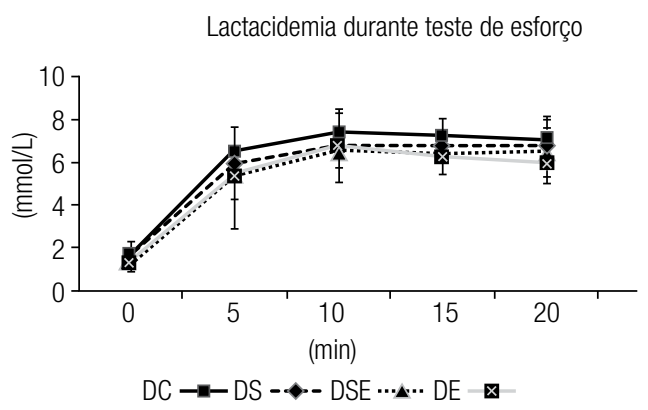

Figura 3. Concentrações de lactato sanguíneo, obtido durante o teste de esforço (sobrecarga equivalente a $5 \%$ do peso corporal), para os grupos diabético controle (DC; $n=7$ ); diabético Spirulina (DS; $n=10)$; diabético exercício (DE; $n=8)$; diabético Spirulina exercício (DSE; $n=11)$. Os dados são expressos em média \pm desvio-padrão. 
A figura 5 apresenta a taxa de remoção de glicose (\%/minuto) obtida durante o TTI. Não foram observadas diferenças significativas entre os grupos.

A glicemia basal $(\mathrm{mg} / \mathrm{dL})$ para todos os grupos, ao final do experimento, foi superior ao critério de inclusão na condição diabética adotado $(\mathrm{DC}=316,1 \pm$ 67,$8 ; \mathrm{DS}=301,4 \pm 118,0 ; \mathrm{DE}=275,4 \pm 80,9 ; \mathrm{DSE}$ $=248,9 \pm 88,2)$. As concentrações de insulina, tanto pancreáticas quanto séricas, ao final do experimento são apresentadas na figura $6 \mathrm{~A} \mathrm{e} \mathrm{B.} \mathrm{Para} \mathrm{as} \mathrm{concen-}$ trações pancreáticas, o grupo DS apresentou concentração estatisticamente menor quando comparado ao grupo DC. Em relação à insulina sérica, a concentração do grupo DS foi significativamente menor ao ser comparada com a dos grupos DE e DES.

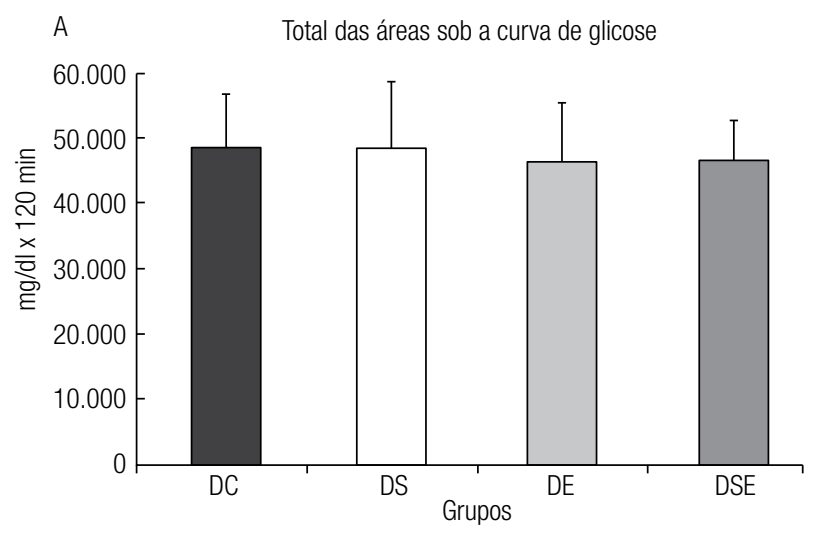

\section{DISCUSSÃO}

O objetivo deste estudo foi analisar os efeitos do exercício aeróbio e da suplementação com Spirulina na homeostase glicêmica de ratos diabéticos. Alguns estudos demonstram os benefícios do exercício $(22,33)$ e outros, os benefícios da Spirulina $(34,35)$ agindo isoladamente no controle glicêmico. No entanto, informações sobre a ação do exercício associado à suplementação de Spirulina no controle glicêmico de ratos diabéticos ainda não tinham sido avaliadas. Os protocolos de tratamento com Spirulina e de treinamento físico aqui empregados não se mostraram eficazes em melhorar a homeostase glicêmica em ratos diabéticos.

No presente estudo, foram utilizados animais apresentando características do diabetes melito

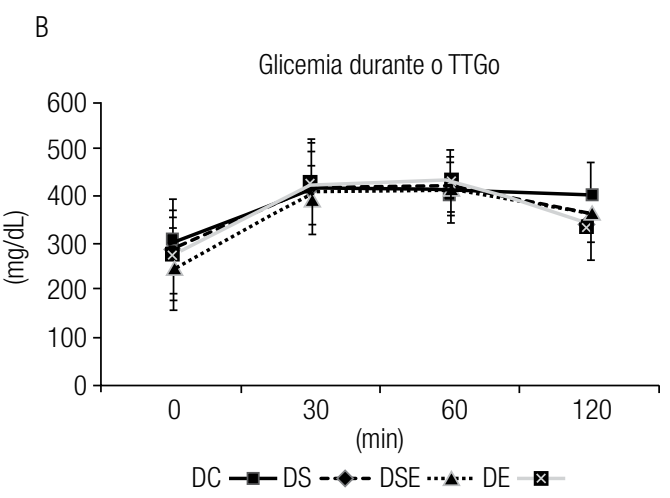

Figura 4. A) Área sob as curvas de glicose e B) Glicemia durante o teste de tolerância à glicose para os grupos diabético controle (DC; $n=6)$; diabético Spirulina (DS; $n=10$ ); diabético exercício (DE; $n=8$ ); diabético Spirulina exercício (DSE; $n=7$ ). Os dados são expressos em média \pm desvio-padrão.

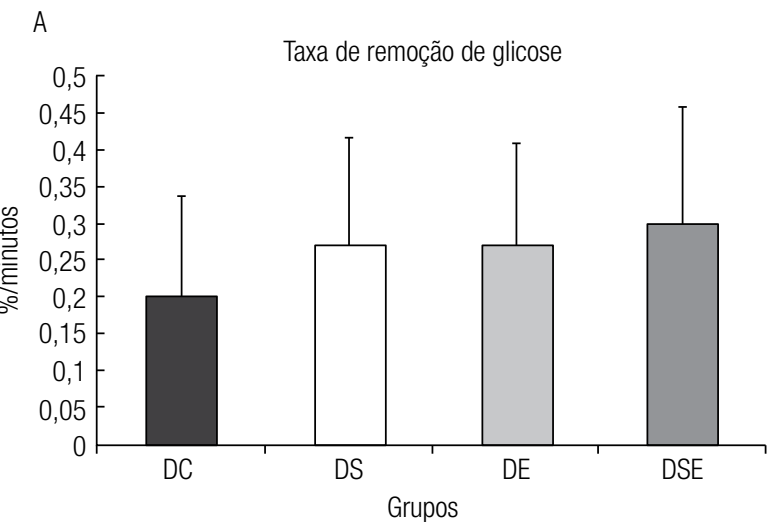

B

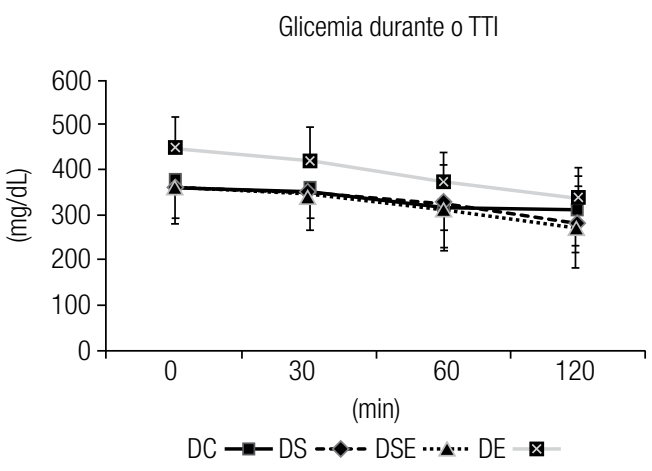

Figura 5. A) Taxa de remoção de glicose e B) Glicemia, obtida durante teste de tolerância à glicose, para os grupos diabético controle (DC; $n=6)$; diabético Spirulina (DS; $n=10$ ); diabético exercício (DE; $n=7$ ); diabético Spirulina exercício (DSE; $n=11$ ). Os dados são expressos em média \pm desvio-padrão. 

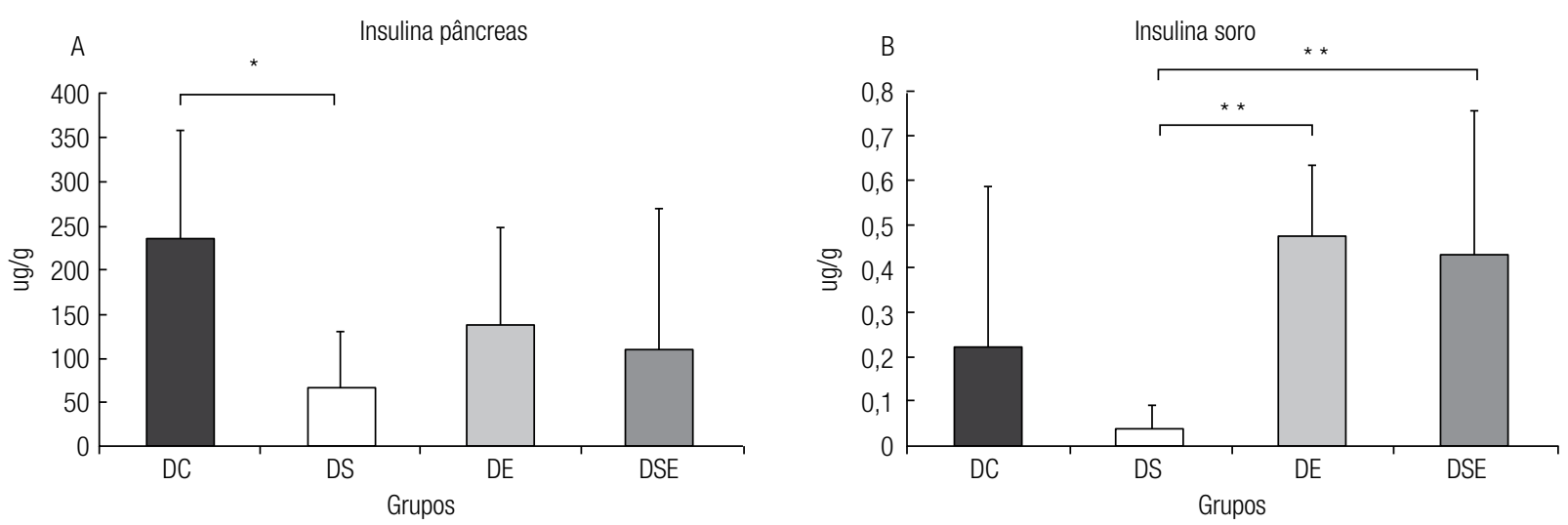

Figura 6. Concentrações de insulina do pâncreas (A) e soro (B), após o período experimental, para os grupos diabético controle $(D C ; A, n=7$ e $B, n=8)$; diabético Spirulina (DS; $A, n=13$ e B, $n=14$ ); diabético exercício (DE; $A, n=9$ e B, $n=9$ ); diabético Spirulina exercício (DSE; $A, n=10$ e B, $n=11$ ). Os dados são expressos em média \pm desvio-padrão. * Diferença significativa $(p<0,05)$ em relação ao grupo DS; ${ }^{* *}$ Diferença significativa $(p<0,05)$ do grupo DS em relação ao DE e DSE.

insulino-dependente e não tratados com insulina. A hipoinsulinemia decorrente dessa doença, além de colaborar com a perda de peso, interfere na ingestão alimentar. A perda de peso ocorre em função da baixa concentração de insulina e isso leva a um estado catabólico de proteínas e gorduras, além de implicar glicosúria associada à poliúria (36). Em relação à ingestão alimentar, o quadro de hipoinsulinemia eleva a secreção dos hormônios orexígenos: neuropeptídeo Y (NPPY) e peptídeos relacionados à cepa agouti, ambos secretados no núcleo arqueado do hipotálamo (37). De acordo com Dong e cols. (38), ratos diabéticos apresentam níveis elevados de NPPY e níveis baixos do hormônio anorexígeno estimulador do $\alpha$-melanócito, contribuindo, assim, para a estimulação da fome. Em nosso estudo não foi encontrada nenhuma diferença entre os grupos devido à ausência do grupo controle contendo ratos sadios. Quando nossos dados são comparados com aqueles do estudo de Moura e cols. (39), que mostraram consumo cerca de duas vezes maior para ratos diabéticos em relação aos controles sadios, observa-se que nossos resultados são similares aos encontrados neste estudo, para os grupos diabéticos.

O protocolo de treinamento físico com natação realizado neste experimento correspondeu a um treinamento aeróbio moderado para ratos diabéticos (27), entretanto, nesta investigação, os grupos exercitados não apresentaram diferenças no metabolismo glicídico advindos do treinamento comparado com seus pares sedentários. Entre as adaptações que o exercício aeróbio proporciona, está a mobilização de substratos energéticos com utilização de oxigênio. $\mathrm{O}$ ácido láctico gerado no metabolismo pode ser convertido novamente em glicose no fígado, mantendo seu nível estabilizado. Em indivíduos submetidos ao exercício físico agudo, quando a demanda energética é elevada e a liberação de lactato na corrente sanguínea extrapola a capacidade de remoção pelos tecidos, grande quantidade desse substrato acumula-se no sangue, precipitando a fadiga por acidose. No teste de esforço, os grupos sedentário e treinado apresentaram estabilização do lactato após dez minutos de exercício, mostrando que ambos realizaram exercício aeróbio e que o metabolismo energético não foi influenciado pelo protocolo de treinamento, pois a cinética do lactato sanguíneo durante o teste foi semelhante em ambos os grupos.

Nos ratos induzidos ao diabetes por aplicação de aloxana, a secreção pancreática de insulina encontra-se prejudicada, o que limita a translocação dos transportadores de glicose GLUT-4 nos tecidos insulinossensíveis. Sabe-se que o exercício ativa a captação muscular da glicose por mecanismos independentes da insulina (40). Além disso, respostas metabólicas ao exercício agudo atuam sobre a captação da glicose após sobrecarga oral. No presente estudo, o protocolo de treinamento utilizado não foi eficaz em melhorar a tolerância à glicose e a sensibilidade periférica à insulina, como mostram os resultados referentes 
à área sob a curva da glicose sérica durante o GTTo e à taxa de remoção de glicose sérica durante o TTI, respectivamente, que foram semelhantes para ratos treinados e sedentários.

De acordo com Moura e cols. (39), que realizaram um estudo envolvendo ratos diabéticos utilizando o mesmo protocolo de treinamento aqui empregado, a intensidade do exercício ( $3,5 \%$ p.c.) pode ter sido ineficiente em melhorar a tolerância à glicose. A ausência de alteração na remoção da glicose sanguínea em decorrência do treinamento era esperada, uma vez que os animais eram considerados insulino-dependentes, em que não se tem resistência ao hormônio e sim redução na produção. Portanto, quando administrada a insulina para todos os grupos, a resposta, na captação de glicose, há de ser a mesma.

No presente estudo, a suplementação de $17 \%$ de Spirulina na dieta não resultou em modificações significativas no comportamento da glicose sérica durante o TTGo e o TTI. São relatados na literatura dados que evidenciam o efeito hipoglicêmico da $S p i$ rulina. Esse efeito é atribuído, principalmente, à fração hidrossolúvel dessa alga que é composta por uma proteína chamada ficocianina que atua como agente hipoglicemiante, e às suas fibras, as quais induzem a redução da absorção de glicose no trato gastrointestinal $(7,18)$. Como ainda é bastante conflitante na literatura a dosagem mais adequada e o tempo de intervenção para a melhor resposta do organismo à alga, isso pode ter influenciado em nossos resultados.

Em concordância com nosso estudo, Lee e cols. (35), avaliando o efeito da suplementação de 12 semanas de Spirulina em pacientes diabéticos (glicemia maior que $126 \mathrm{mg} / \mathrm{dL}$ ), apesar dos resultados favoráveis em relação aos aspectos dos perfis lipídico, antioxidativo e antiinflamatório, não encontraram alterações significativas na glicose e insulina do soro. Em um estudo realizado por Parikh e cols. (34), em que foram utilizados $2 \mathrm{~g} /$ dia de suplementação de Spirulina durante dois meses, apesar de não significantes, valores reduzidos da glicemia de pacientes diabéticos foram encontrados.

De forma geral, nossos resultados, mostrando poucos efeitos metabólicos da Spirulina e do exercício em ratos diabéticos insulino-dependentes, podem ser explicados, em parte, pelo curto período de intervenção. Seis semanas de tratamento podem não ter sido suficientes para induzir adaptações metabólicas que pudessem levar a uma atenuação do quadro de hiperglicemia, tais como estímulo da secreção de insulina, uma vez também que o diabetes melito tipo 1 é caracterizado pela destruição das células $\beta$ pancreáticas.

Em suma, os protocolos de exercício e de suplementação com Spirulina empregados no presente estudo não foram suficientes para promover melhora na homeostase glicêmica de ratos diabéticos.

Agradecimentos: os autores agradecem o indispensável apoio dos técnicos do Laboratório de Biodinâmica do Departamento de Educação Física da Unesp, Campus Rio Claro, Clarice Sibuya e José Roberto Rodrigues, o Conselho Nacional de Desenvolvimento Científico e Tecnológico (CNPq) e a Fundação de Amparo à Pesquisa do Estado de São Paulo (Fapesp) pelo suporte financeiro. Agradecem também a Corn Products Brasil ${ }^{\circledR}$ pela doação de materiais para produção das dietas usadas no presente experimento.

Declaração: os autores declaram não haver conflitos de interesse científico neste estudo.

\section{REFERÊNCIAS}

1. Wild S. Global prevalence of diabetes, estimates for the year 2000 and projections for 2030. Diabetes Care. 2004;27:1047-053.

2. Das AV, Padayutti PS, Paulose CS. Effect of leaf extract of Aegle marmelose (L) Corra ex Roxb. On histological and ultrastructural changes in tissues of streptozotocin induced diabetic rats. Indian J Exp Biol. 1996;14:341-4.

3. Garcia MJ, MCnamara PM, Gordan T, Kannel WB. Morbidity and mortality in diabetics in the Framingham population. Sixteen year follow-up study. Diabetes. 1974;23(2):105-12.

4. Holman RR, Turner RC. Oral agents and insulin in the treatment of NIDDM. In: Pickup J, Williams G (eds.). Blackwell, Oxford:Text Book of Diabetes; 1991. p. 467-9.

5. Goksel G, Mehmet ZH. Evaluation of antidiabetic, antioxidant and vasoprotective effects of Posidonia oceanica extract. J Ethnopharmacol. 2008;115:122-30.

6. Tülay B, Utku B, Oya UK, Sinem GU, HasretY. In vivo assessment of antidiabetic and antioxidant activities of rosemary (Rosmarinus officinalis) in alloxan-diabetic rabbits. J Ethnopharmacol. 2008;116:64-73.

7. Ambrosi MA, Reinehr CO, Bertolin TE, Costa JAV, Colla LM. Propriedades de saúde de Spirulina spp. Rev Ciênc Farm Básica Apl. 2008;29(2):109-17.

8. Belay A, Ota Y, Miyakawa K, Shimatsu H. Current knowledge on potential health benefits of spiruline. J Appl Physic. 1993;5:235-41.

9. Nakaya N, HonmaY, Goto Y. Cholesterol lowering effect of Spirulina. Nutr Rep Int 1988;37:1329-37.

10. Furst PT. Spiruline - a nutritious alga, once, a staple of Aztec diet, could feed many of the words hungry people. Human Nature. 1978;60:459-70.

11. Kay RA. Microalgae as food and supplement. Crc Cr Rev Food Sci. 1991;30(6):555-73.

12. Kappor R, Mehta U. Supplementary effect of spiruline on hematological status of rats during pregnancy and lactation. Plant Foods Hum Nutr Aliment. 1998;29(6):517-34.

13. Voltarelli FA, Mello MAR. Spirulina enhanced the skeletal muscle protein in growing rats. Eur J Nutr. 2008;47(7):393-400. 
14. Combs W, Sonis ST, Fitzgerald J, Tracy C, Wilson R. In vivo and in vitro effects of beta carotone and algae extracts in murine tumor models. Nutr Cancer. 1989;12:371.

15. Alves CR, Voltarelli FA, Mello MAR. Spirulina como fonte protéica na recuperação nutricional de ratos: efeitos sobre o músculo esquelético. Lecturas: Ed Fis y Dep. 2005;10(86):1-11.

16. Becker EW, Jakober R, Luft D, Schmülling RM. Clinical and biochemical evaluation of the alga Spirulina with regard to its application in the treatment of obesity. A double-blind cross-over study. Nutr Rep Int. 1986;33:565-72.

17. Hernández AR, Castillo JLB, Oropeza MAJ, Zagoya JCD. Spirulina maxima prevents fatty liver formation in CD-1 male and female mice with experimental diabetes. Life Sci. 2001;69:1029-37.

18. HosoyamadaY, Takai T, Kato T. Effects of water-soluble and insoluble fractions of Spirulina on serum lipid components and glucose tolerance in rats. J Jap Soc Nutr Food Sci. 1991;44:273-7.

19. De Caire GZ, De Cano MS, De Mule CZ. Steyerthal N, Piantanida M. Effect of Spirulina platensis on glucose, uric acid and cholesterol levels in the blood of rodents. Int J Exp Bot. 1995;57:93-6.

20. Layam A, Reddy CLK. Antidiabetic property of spirulina. Diabetologia Croat. 2006;35(2):29-33.

21. Ivy JL, ZdericTD, Fogt DL. Prevention and treatment of non-insulin-dependent diabetes mellitus. Exercise Sport Sci R. 1999;27:1-35.

22. Luciano E, Mello MAR. Atividade física e metabolismo de proteínas em músculo de ratos diabéticos experimentais. Rev Paul Educ Fís. 1998;2(12):202-9.

23. Lenzen $\mathrm{S}$. The mechanisms of alloxan- and streptozotocin-induced diabetes. Diabetologia. 2008;2:216-26.

24. American Diabetes Association. Physical activity/exercise and diabetes. Diabetes Care. 2004;27(III):S58-S62.

25. Reeves PG, Nielsen FH, Fahey GC. AIN-93 purified diets for laboratory rodents: final report of the American Institute of Nutrition AdHoe Writing Committee on the Reformulation of AIN-76A. J Nutr. 1993;123:1938-51.

26. Voltarelli FA, Gobatto CA, Mello MAR. Determination of anaerobic threshold in rats using the lactate minimum test. Braz $\mathrm{J}$ Med Biol Res. 2002;35:1389-94.

27. Oliveira CAM, Luciano E, Marcondes MCCG, Mello MAR. Effects of swimming training at the intensity equivalent to aerobic/ana- erobic metabolic transition in alloxan diabetic rats. J Diabetes Complicat. 2007;21:258-64.

28. Mathews JNS, Altman DG, Campbell MJ, Royston P. Analysis of serial measurements in medical research. Br Med J. 1990;27:230-5.

29. Lundbaeck K. Intravenous glucose tolerance test as a tool in definition and diagnosis of diabetes mellitus. Br Med J. 1962;2:1507-13.

30. Engels RC, Jones JB. Causes and elimination of erratic blanc in enzymatic metabolic assays involving the use of NAD in alkaline hydrazine buffers: improved conditions for assay of L-glutamate, L-lactate and other metabolites. Anal Biochem. 1978;88:475-84.

31. Herbert V, Lau KS, Gottlieb CW, Bleicher SJ. Coated charcoals immunoassay of insulin. J Clin Endocrinol Metab. 1965;25:1375-84.

32. Carpinelli AR. Estudo da secreção de insulina e da homeostase glicêmica em ratos desnutridos [dissertação]. Instituto de Ciências Biomédicas, Universidade de São Paulo, São Paulo; 1978.

33. Khawali C, Andriolo A, Ferreira SRG. Benefícios da atividade física no perfil lipídico de pacientes com diabetes tipo 1. Arq Bras Endocrinol Metabol. 2003;47(1):49-54.

34. Parikh $P$, Mani $U$, lyer $U$. Role of spirulina in the control of glycemia and lipidemia in type 2 diabetes mellitus. J Med Food. 2001;4(4):193-9.

35. Lee EH, Park JE, Choi YJ, Huh KB, Kim WY. A randomized study to establish the effects of spirulina in type 2 diabetes mellitus patients. Nutr Res Pract. 2008;2(4):295-300.

36. Kimball SR, VaryTC, Jefferson LS. Regulation of protein synthesis by insulin. Annu Rev Physiol. 1994;56:321-48.

37. Asakawa A, Toyoshima M, Inoue K, Koizume A. Ins2Akita mice exhibit hyperphagia and anxiety behavior via the melanocortin system. Int J Mol Med. 2007;19:649-52.

38. Dong J, Peeters TL, De Smet B, Moechars D, Delporte C, Vanden Berghe $P$, et al. Role of endogenous ghrelin in the hyperphagia of mice with streptozotocin-induced diabetes. Endocrinology. 2006;147:2634-42.

39. Moura LP, Chyoda A, Teixeira CVL, Sponton CHG, Coelho FGM, Lima MCS, et al. Effects of physical exercise and cinnamon extract on blood chemistry of type 1 diabetic rats. J Exerc Physiol. 2010;13(4):17-28.

40. Machado UB, Schaan BF, Seraphim PM. Transportadores de glicose na síndrome metabólica. Arq Bras Endocrinol Metabol. 2006;50(2):177-89. 